\title{
Seqüestro pulmonar: uma série de nove casos operados"
}

\author{
Paulo M. PÊgo-Fernandes ${ }^{1}$, Cibele Hasmann Freire ${ }^{2}$, FAbio Biscegli Jatene ${ }^{1}$, \\ Ricardo Beyruti ${ }^{3}$, Francisco VARgas Suso ${ }^{4}$, SÉrgio Almeida de Oliveira ${ }^{5}$
}

Seqüestro pulmonar é uma anomalia congênita que envolve parênquima e vascularização pulmonar e apresenta-se como extralobar ou intralobar. Objetivo: Descrever os casos de seqüestro pulmonar tratados no InCor e Hospital das Clínicas da FMUSP no período de 1987 a 1996. Método: Análise retrospectiva dos prontuários. Resultados: Foram tratados nove pacientes, sendo quatro mulheres e cinco homens; duas crianças e sete adultos. Infecção respiratória de repetição e hemoptise foram achados clínicos freqüentes nesses pacientes. Todos os casos eram intralobares. A principal localização

foi no lobo inferior esquerdo (66\%). Apenas um diagnóstico foi intra-operatório. Nos outros oito casos, o diagnóstico foi suspeitado pela radiografia de tórax (100\%) e confirmado pela arteriografia

(77\%) e/ou tomografia computadorizada (66\%). Lobectomia (77\%) foi o principal tratamento cirúrgico, com baixa morbidade pós-operatória e sem mortalidade. Exame anatomopatológico foi realizado em sete casos e confirmou a doença. Conclusões: O seqüestro pulmonar é uma entidade incomum, em que a tomografia computadorizada e a arteriografia são os exames que mais informações oferecem para um diagnóstico definitivo e seguro. A ressecção do tecido envolvido leva a excelentes resultados. (J Pneumol 2002;28(4):175-179)

\section{Pulmonary sequestration: a series of nine cases operated on}

Background: Pulmonary sequestration is a congenital anomaly involving the pulmonary parenchyma and its vascularization, and it presents as extralobar or intralobar. Aim: Describe the cases treated at the Institute of Heart (InCor) and Clinical Hospital of the Medical School of the

University of São Paulo (FMUSP). Method: Retrospective analyses of the files of the patients treated over the 10-year period taking demographic, clinical and laboratorial data. Results: The patients were recurrent pulmonary infection and hemoptysis were frequent clinical findings in these patients. All cases were intralobar. The main localization was the left lower lobe (66\%). Only one diagnosis was made intra-operatively. In the other eight cases, diagnosis was suggested on chest radiographs (100\%) and confirmed by angiography (77\%) and computed tomography

(66\%). Lobectomy (77\%) was the most common surgical treatment, with low post-operative morbidity, and no mortality. Anatomic and histologic examination was performed in seven cases and confirmed the pathology. Conclusion: Pulmonary sequestration is an uncommon entity, in

which the computed tomography and the angiography are the radiological investigations that provide the most information to a definitive and secure diagnosis. Resection of the involved tissue leads to excellent results.

Descritores - Radiografia. Angiografia. Tomografia computadorizada por raios X. Artéria pulmonar. Pneumonectomia. Pneumopatias.

Key words - Radiography. Angiography. CT scan by X-rays. Pulmonary artery. Pneumonectomy. Lung diseases.
Siglas e abreviaturas utilizadas neste trabalho

SPE - Seqüestro pulmonar extralobar

SPI - Seqüestro pulmonar intralobar
* Trabalho realizado na Faculdade de Medicina da Universidade de São Paulo (FMUSP).

1. Professor Livre-Docente do Departamento de Cardiopneumologia.

2. Aluno do $4^{\circ}$ ano de Graduação do Departamento de Medicina da Universidade de Taubaté.

3. Médico Assistente do Serviço de Cirurgia Torácica.

4. Professor Titular da Disciplina de Pneumologia.

5. Professor Titular da Disciplina de Cirurgia Torácica e Cardiovascular.

J Pneumol 28(4) - jul-ago de 2002
Endereço para correspondência - Paulo M. Pêgo-Fernandes, Instituto do Coração-HC-FMUSP, Av. Dr. Enéas de Carvalho Aguiar, 44, 2 andar - 05403-000 - São Paulo, SP. Tel./fax: (11) 3069-5248; email: paulopego@incor.usp.br

Recebido para publicação em 21/12/01. Aprovado, após revisão, em 30/4/02. 


\section{INTRODUÇÃO}

Hubber, em 1777, descreveu uma artéria anômala saindo da aorta para suprir um lobo inferior direito normal. Rokitansky (1861) e também Rektorzik (1861) descreveram casos que pareciam ser seqüestro pulmonar extralobar. Pryce, em 1946, introduziu o termo seqüestro pulmonar e definiu a anatomia ${ }^{(1)}$.

Seqüestro pulmonar é uma malformação congênita caracterizada por uma massa de tecido pulmonar não funcionante separada da árvore traqueobrônquica normal e vascularizada por uma artéria sistêmica anômala, comumente derivada da aorta torácica, do tronco celíaco ou das artérias intercostais. Essa anomalia representa entre 0,15 e $6,45 \%$ de todas as malformações pulmonares. Nenhuma anormalidade cromossômica foi identificada nos pacientes que apresentavam seqüestro pulmonar. Dois tipos de seqüestro pulmonar são reconhecidos, dependendo da presença ou não de envoltório próprio ${ }^{(2)}$.

$\mathrm{O}$ seqüestro pulmonar extralobar (SPE) está encerrado dentro de sua própria membrana pleural, em geral, em íntima proximidade com o parênquima pulmonar. Recebe seu suprimento arterial da aorta abdominal ou de um de seus ramos e a drenagem é feita geralmente para veia ázigos, hemiázigos ou porta, criando uma derivação da esquerda para a direita. Não contém espaços aéreos. É geralmente diagnosticado na infância ou adolescência, sem evidência de infecção ${ }^{(1-5)}$.

O seqüestro pulmonar intralobar (SPI) é definido como um segmento do parênquima pulmonar situado dentro do revestimento pleural normal do pulmão, não conectado à árvore traqueobrônquica e suprido por circulação arterial sistêmica anômala. Deriva seu suprimento arterial, quase invariavelmente, da aorta ou de um de seus ramos. Os lobos superiores são raramente afetados; nesses casos o vaso anômalo origina-se comumente da aorta torácica ascendente ou de um de seus principais ramos, sendo muito comuns outras anomalias cardíacas. No SPI a artéria nutriz é geralmente de largo calibre. A drenagem venosa é feita por veias pulmonares, produzindo uma derivação da esquerda para a esquerda, porém há casos relatados de drenagem para a veia cava inferior ou para o sistema ázigos. Normalmente, não tem comunicação com a árvore traqueobrônquica e, quando isso ocorre, está geralmente associada com infecção no pulmão seqüestrado, provavelmente causada por erosão e fistulização. Sessenta a noventa por cento dos seqüestros estão localizados no tórax póstero-inferior esquerdo e lobo inferior. O segmento póstero-basal do lobo inferior esquerdo é local comum para SPI, mas tem sido identificado ainda em lobos superiores e, raramente, em lobo médio ou bilateralmente $e^{(1-4)}$.

Classicamente, o tratamento de seqüestro pulmonar é cirúrgico. O objetivo deste estudo é a análise da experiência do Serviço de Cirurgia Torácica da FMUSP, no tratamento cirúrgico de nove pacientes.

\section{PACIENTES E MÉTODOS}

Foi feita uma análise retrospectiva dos casos de seqüestro pulmonar submetidos a tratamento cirúrgico no Instituto do Coração e Hospital das Clínicas da FMUSP, no período de 1987 a 1996.

Foram analisados nove pacientes, sendo cinco do sexo masculino e quatro do feminino. Dois pacientes eram crianças com idade entre sete e 13 anos, e sete adultos, com idade variando entre 24 e 37 anos, com média de 31,5 anos (Tabela 1 ).

Todos os pacientes apresentaram história de sintomas respiratórios, principalmente hemoptise e infecção respiratória de repetição, além de tosse, dispnéia e broncossupuração (Tabela 1 ).

Infecção respiratória de repetição (quatro casos), broncossupuração (dois), hemoptise (dois) e associação de infecção de repetição e broncossupuração (um) foram as principais indicações cirúrgicas.

TABELA 1

Idade, sexo e quadro clínico dos pacientes analisados

\begin{tabular}{|c|c|c|c|}
\hline Paciente & Idade & Sexo & Sintomas \\
\hline 1 & 7 & $\mathrm{~F}$ & Infecção respiratória de repetição \\
\hline 2 & 13 & $\mathrm{~F}$ & Infecção respiratória de repetição, dispnéia \\
\hline 3 & 37 & M & Infecção respiratória de repetição, tosse \\
\hline 4 & 37 & M & Broncossupuração \\
\hline 5 & 34 & $\mathrm{~F}$ & Diagnóstico durante internação por artrite reumatóide \\
\hline 6 & 36 & $\mathrm{~F}$ & Febre, tosse, broncossupuração, infecção respiratória de repetição, dispnéia \\
\hline 7 & 27 & M & Tosse, escarro, dispnéia, pleurite, infecção respiratória de repetição \\
\hline 8 & 26 & M & Hemoptise \\
\hline 9 & 24 & M & Hemoptise \\
\hline
\end{tabular}




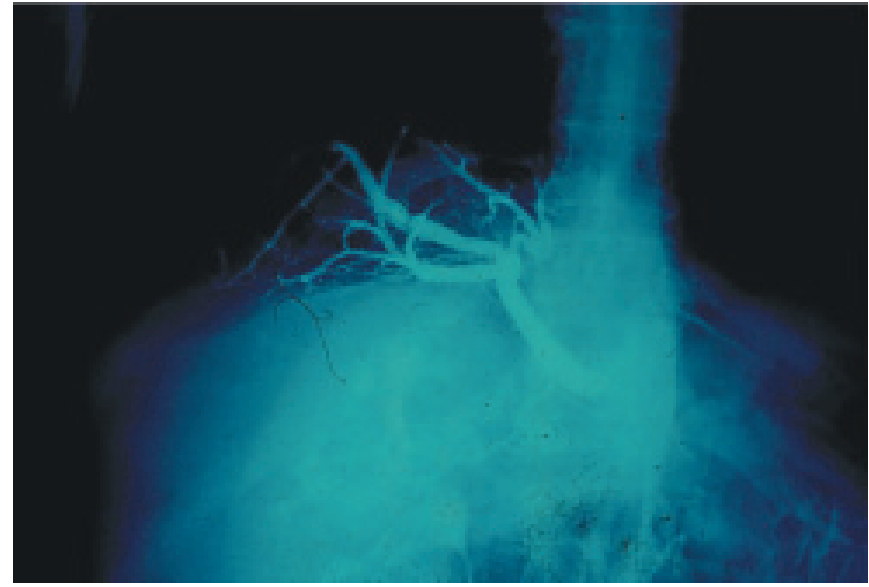

Figura 1 - Arteriografia mostrando a artéria sistêmica anômala em direção ao lobo inferior direito

A pesquisa de BAAR no escarro foi realizada em cinco casos, nos quais foi negativa. A cultura de secreção respiratória foi feita em seis pacientes, dos quais duas não apresentaram crescimento bacteriano. Dentre as que foram positivas, as bactérias encontradas foram Streptoccocus viridans, Branhamella catarrhalis, Haemophilus sp., Enterobacter sp., Pseudomonas aeruginosa e Serratia marcescens.

Apenas um diagnóstico ocorreu no intra-operatório (criança, sete anos). Os oito casos restantes foram diagnosticados no pré-operatório, sendo estes todos de SPI.

Quanto aos métodos diagnósticos: radiografia de tórax foi realizada em $100 \%$ dos pacientes; $77 \%$ foram submetidos a arteriografia (Figura 1) e 66\% a tomografia computadorizada. Os outros métodos diagnósticos (ressonância magnética, ultra-sonografia, cintilografia, broncografia e planigrafia) poucas vezes foram usados (Tabela 2).

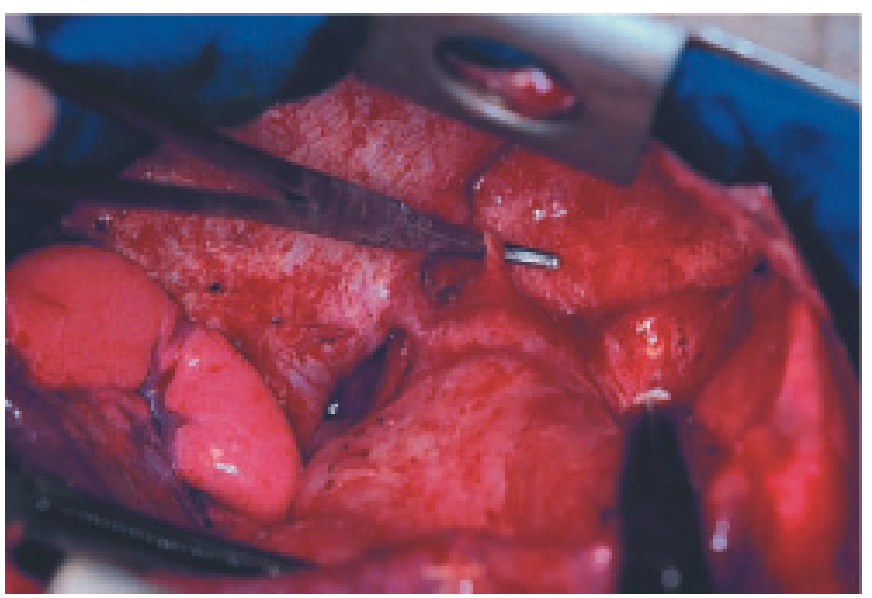

Figura 2 - Foto da operação evidenciando a artéria anômala

Todos os casos foram de seqüestro pulmonar intralobar. Oito estavam localizados em lobos inferiores, sendo seis à esquerda e dois à direita. Seqüestro no lobo superior ocorreu somente uma vez e à direita (Tabela 2).

Quanto ao tratamento cirúrgico, foi realizada lobectomia em sete casos (Figura 2) e duas segmentectomias (Tabela 2).

\section{Resultados}

O diagnóstico anatomopatológico teve resultados compatíveis com seqüestro pulmonar.

Nenhuma malformação associada foi relatada nos casos estudados.

Dois pacientes apresentaram complicações pós-operatórias, sendo que em um ocorreu discreta tosse com expectoração hemoptóica e, em outro, empiema.

TABELA 2

Tipo de seqüestro pulmonar, localizações, exames diagnósticos e tratamento cirúrgico realizado nos pacientes

\begin{tabular}{cllll}
\hline Paciente & Tipo & Local & \multicolumn{1}{c}{ Exames } & \multicolumn{1}{c}{ Operação } \\
\hline 1 & SPI & LIE & RX & Lobectomia \\
2 & SPI & LIE & RX, TC, ART, RM & Lobectomia \\
3 & SPI & LID & RX, TC, ART & Lobectomia \\
4 & SPI & LIE & RX, TC, BR, PL & Lobectomia \\
5 & SPI & LIE & RX, TC, ART, US, PL & Segmentectomia posterior basilar \\
6 & SPI & LID & RX, ART, BR & Segmentectomia basilar \\
7 & SPI & LIE & RX, TC, ART & Lobectomia \\
8 & SPI & LSE & RX, ART & Lobectomia \\
9 & SPI & LIE & RX, TC, ART, PL & Lobectomia \\
\hline
\end{tabular}

SPI - seqüestro pulmonar intralobar; LIE - lobo inferior esquerdo; LID - lobo inferior direito; LSE - lobo superior esquerdo; RX - radiografia de tórax; TC - tomografia computadorizada; ART - arteriografia; RM - ressonância magnética; BR - broncografia; PL - planigrafia; US - ultra-sonografia. 
Não houve óbitos precoces ou tardios.

Quanto à evolução tardia desses pacientes, não houve relatos de recidivas de problemas respiratórios.

\section{DISCUSSÃO}

O seqüestro pulmonar é uma doença pulmonar rara que pode apresentar-se em duas variedades: extralobar $e$ intralobar. A distribuição da entidade intralobar é praticamente igual entre o sexo masculino e o feminino, sendo, geralmente, diagnosticada após os 20 anos como resultado de pneumonia aguda, mais freqüentemente na terceira década de vida ${ }^{(2-5)}$. Neste estudo não foi verificada variação significativa entre os sexos e o SPI foi mais incidente em adultos, com idade média de 31,5 anos. Em estudo semelhante de Louie et al., em 1993, a idade média dos pacientes analisados foi de 32 anos $^{(6)}$.

Tipicamente, o SPI é revelado por sintomas secundários de infecção respiratória ou problemas cardíacos ${ }^{(7)}$. Hemoptise, dispnéia, cianose e outros sinais de insuficiência respiratória são freqüentemente encontrados nesses pacientes ${ }^{(1)}$. A hemoptise é comum e geralmente descrita como sangramento vivo e abundante, podendo ser superior a $800 \mathrm{ml}^{(5)}$. O paciente com SPI geralmente tem história de pneumonia de repetição na infância, porém isso não é obrigatório para confirmação diagnóstica ${ }^{(6)}$. Os sintomas cardíacos causados pela derivação da esquerda para a direita estão diretamente relacionados com o volume do fluxo e são, portanto, dependentes do calibre do vaso anômalo que irriga o seqüestro. Assim como nos estudos de outros autores ${ }^{(2,6,7)}$, os principais sintomas observados em nossa análise e que foram causas de indicação cirúrgica foram infecção respiratória de repetição, broncossupuração e hemoptise.

Apesar de incomum, o alto fluxo sanguíneo proveniente de um vaso da aorta pode levar a diagnóstico errôneo de doença valvular cardíaca primária ${ }^{(8)}$. O diagnóstico diferencial inclui cisto pulmonar congênito ou adquirido, tumores de mediastino posterior, hérnia hiatal de Boshdalek e cisto diafragmático congênito(7).

Raramente, são encontrados dados bacteriológicos em relatos de casos; no entanto, é freqüente encontrarmos pesquisa de bacilo álcool-acidorresistente $e^{(4,5)}$. Nesse estudo seis pacientes realizaram cultura de secreção respiratória e quatro apresentaram resultado positivo; pesquisa de BAAR foi realizada em cinco casos, todos negativos. Em estudo semelhante de Halkic et al. ${ }^{(2)}$, os microrganismos relatados foram Mycobacterium tuberculosis, Aspergillus umigatus e Nocardiose asteroides, portanto, diferentes dos encontrados em nossos resultados.

A radiografia de tórax é um dos principais exames para diagnóstico de seqüestro pulmonar, sendo necessária a confirmação pela arteriografia e tomografia computado- rizada $^{(2,5)}$, como observado em nosso estudo. Na radiografia simples de tórax a aparência do SPI é geralmente de massa radiopaca ou densidade de parênquima difusa. Cistos contendo ar com ou sem nível líquido podem estar presentes. A arteriografia é o método mais seguro para diagnóstico pré-operatório de seqüestro pulmonar porque pode demonstrar a artéria sistêmica anômala que supre a lesão(7). Mais recentemente, o ultra-som Doppler demonstrou os ramos aórticos anômalos sem a morbidade (anestesia geral e risco de injúria arterial ou trombose) da arteriografia ${ }^{(1)}$. A tomografia tem sido usada para demonstrar características anormais do parênquima e pode identificar o suprimento sanguíneo aberrante do pulmão seqüestrado(6), mas para confirmação definitiva do diagnóstico prefere-se a arteriografia(2). A angiografia préoperatória não só confirma o diagnóstico, mas demonstra com precisão a origem do suprimento vascular, informação esta importante para o sucesso da operação. A transecção acidental da artéria sistêmica anômala pode levar a conseqüências fatais; portanto, é necessário identificar e ligar a artéria para iniciar a ressecção(1,5,7). Nesta série, o único caso em que o diagnóstico foi intra-operatório, o paciente (criança, sete anos) havia feito apenas radiografia de tórax. Dois casos em que foi realizada arteriografia após a radiografia não houve necessidade de submeter o paciente a tomografia computadorizada, pois o diagnóstico foi definitivo, permitindo inclusive o reconhecimento seguro da artéria anômala. Halkic et al.(2) estudaram 26 casos de seqüestro pulmonar, sendo que 20 pacientes foram diagnosticados pela radiografia de tórax e confirmados pela arteriografia.

O seqüestro intralobar é três vezes mais freqüente do que a variedade extralobar ${ }^{(4)}$. Halkic et al. ${ }^{(2)}$, estudando 26 casos de seqüestro pulmonar, relataram $73 \%$ do tipo intralobar. Louie et al. ${ }^{(6)}$, em uma análise de 10 casos, constataram 50\% do tipo intralobar; $90 \%$ do total de casos estavam localizados em lobo inferior. Tsolakis et al. ${ }^{(7)}$, analisando oito casos, descreveram $75 \%$ do tipo intralobar; $100 \%$ dos casos estavam localizados em lobo inferior. Em nossa análise de nove pacientes, relatamos 100\% do tipo intralobar e em $89 \%$ (oito pacientes) dos casos estavam localizados em lobo inferior, sendo que destes, seis eram do lado esquerdo, característica freqüentemente observada ${ }^{(1,2,4)}$.

O tratamento para seqüestro pulmonar é a ressecção do parênquima pulmonar seqüestrado, com o específico acesso, dependendo da localização da lesão. Esse tipo de operação usualmente não é difícil nos casos de seqüestro extralobar, já que a malformação está bem separada do pulmão normal pelo seu próprio envelope pleural(9). No seqüestro intralobar, entretanto, alterações inflamatórias resultantes de infecções prévias podem complicar a operação devido à destruição do plano intersegmental. A lo- 
bectomia, e não apenas a ressecção isolada do segmento seqüestrado, é geralmente o tratamento de escolha ${ }^{(1,9)}$. Nesta série, a lobectomia foi realizada em $77,8 \%$ dos casos. A mesma predominância foi observada nos estudos de Halkic et al. ${ }^{(2)}$ e Tsolakis et al. ${ }^{(7)}$. Segmentectomia tem sido sugerida se o tecido ao redor estiver intacto; no entanto, pode resultar em infecções recorrentes e necessitar lobectomia ${ }^{(7)}$. Nos casos em que realizamos a segmentectomia o resultado também foi satisfatório. Em nenhum desses estudos ${ }^{(2,6)}$ houve secção inadvertida da artéria, demonstrando novamente a importância de diagnóstico pré-operatório seguro. O tratamento cirúrgico é desaconselhado por alguns autores no caso de seqüestro pulmonar intralobar, sendo indicado apenas em caso de infecção. A simples ligadura do vaso anômalo previne a hemoptise, mas não a infecção.

Há poucos relatos na literatura de realização de cirurgia torácica videoassistida ${ }^{(10,11)}$ para tratamento do seqüestro pulmonar. Entretanto, deve-se enfatizar a importância de análise pré-operatória detalhada das artérias anômalas pela arteriografia seletiva, para que haja manipulação segura durante a toracoscopia.

A ressecção cirúrgica do seqüestro pulmonar é um procedimento que acarreta poucas complicações no pós-ope-

\section{REFERÊNCIAS}

1. Quaglia MP. Congenital anomalies. In: Pearson FG, editor. Thoracic surgery. New York: Churchill Livingstone, 1995;411-32.

2. Halkic N, Cuénoud PF, Corthésy ME, Ksontini R, Boumghar M. Pulmonary sequestration: a review of 26 cases. Eur J Cardiothorac Surg 1998; 14:127-33.

3. Fraser RG, Paré JA. Diagnóstico das doenças do tórax. In: Anomalias pulmonares de origem evolutiva. $2^{\underline{a}}$ ed. São Paulo: Manole, 1981; 596-643.

4. Mady C, Abensur H, Jatene F, Barreto ACP, Bellotti G, Pileggi F. Seqüestro intralobar pulmonar. Relato de caso e revisão da literatura. Arq Bras Cardiol 1987;49:101-4.

5. Scully RE, Galdabini JJ, Mcneely BU. Case records of the Massachusetts General Hospital. Case 18-1981. N Engl J Med 1981;304:10906.

6. Louie HW, Martin SM, Mulder DG. Pulmonary sequestration: 17-year experience at UCLA. Am Surg 1993;59:801-5. ratório, como observado em nossa análise. As complicações observadas pelo estudo de Halkic et al. ${ }^{(2)}$ ocorreram após operação de seqüestro extralobar, incluindo, principalmente, hemotórax e empiema.

Ao exame anatomopatológico o seqüestro pulmonar apresenta-se como massa cística ou sólida que contém constituintes normais do pulmão, como músculo liso, epitélio brônquico e cartilagem, mas ausência da estrutura brônquica e, na maioria dos casos, não existe comunicação com o tecido pulmonar vizinho(5).

Assim como no estudo de Halkic et al. ${ }^{(2)}$ e Louie et al. ${ }^{(6)}$, a evolução tardia dos pacientes operados foi excelente, confirmando um resultado altamente favorável para tratamento cirúrgico de seqüestro pulmonar intralobar.

Como considerações finais, o seqüestro pulmonar é uma anomalia congênita de difícil diagnóstico devido a sua raridade e, muitas vezes, reconhecida somente na vida adulta. O diagnóstico precoce é de extrema importância para prevenção de infecções respiratórias de repetição, muitas vezes, refratárias ao tratamento com antibióticos. A tomografia computadorizada e a arteriografia são os exames que mais se aplicam a um diagnóstico definitivo $e$ seguro. O tratamento clássico pela ressecção cirúrgica propicia bons resultados.

7. Tsolakis CC, Kollias VD, Panayotopoulos PP. Pulmonary sequestration: experience with eight consecutive cases. Scand Cardiovasc J 1997; 31:229-32.

8. Kimbrell B, Degner T, Glatleider P, Applebaum H. Pulmonary sequestration presenting as mitral valve insufficiency. J Pediatr Surg 1998; 33:1648-50

9. Van Raemdonck D, De Boeck K, Devlieger H, Demedts M, Moerman $\mathrm{P}$, Coosemans W, et al. Pulmonary sequestration: a comparison between pediatric and adult patients. Eur J Cardiothorac Surg 2001;19: 388-95.

10. Nakamura H, Makihara K, Taniguchi Y, Ishiguro K, Ohgi S. Thoracoscopic surgery for intralobar pulmonary sequestration. Ann Thorac Cardiovasc Surg 1999;5:405-7.

11. Della Porta M, Galli A, Rebuffat C. Videothoracoscopic approach in the treatment of intralobar pulmonary sequestration. Report of a case. Chir Ital 2000;52:299-302. 